\title{
Growth Inhibition of Shenfoweikang Herbs on Gastric Carcinoma Via Signal Transduction Pathways
}

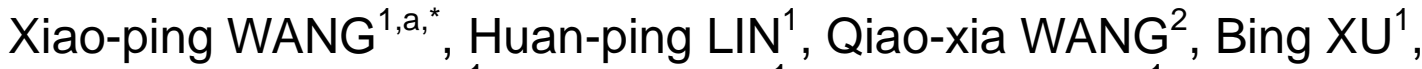 \\ Xuan QU ${ }^{1}$, Bao-ning $\mathrm{QI}^{1}$ and $\mathrm{Na}^{\mathrm{CH}} \mathrm{HNG}^{1}$ \\ ${ }^{1}$ Key Laboratory of Molecular Biology and Pathology, Shaanxi University of Chinese \\ Medicine, Xianyang, Shaanxi 712046, PR China \\ ${ }^{2}$ Department of Infectious Diseases, Xi'an Central Hospital, Xi'an, Shaanxi 710000, \\ PR China \\ awxpphd@aliyun.com \\ ${ }^{*}$ Corresponding author
}

Keywords: Shenfoweikang, Mechanism, Signal Transduction Pathways, Gastric Carcinoma.

\begin{abstract}
To verify the anti-tumor effect of the Shenfoweikang on gastric cancer, $\mathrm{BALB} / \mathrm{C}$ mice grafted with a mouse gastric adenocarcinoma cell line MFC was used as the experimental model. The mice received Shenfoweikang herbs over a 60 -day period starting at the first day. The tumor size was periodically measured. Apoptotic indices (AI) were examined by the TUNEL method and flow cytometric analysis. The expression of VEGF, STAT3 and DEC1 in tumor tissues was examined by immunostaining. Compared with the control group, tumor growth in the mice treated with Shenfoweikang decoction were significantly inhibited $(P<0.05)$. AI in the mice was significantly increased after the Shenfoweikang decoction treatment. The expressions of VEGF, STAT3 and DEC1 in tumor tissues were down-regulated after treated with Shenfoweikang decoction. The anti-tumor effect of Shenfoweikang herbs is related with the induction of the cell apoptosis and down-regulation of VEGF, STAT3 and DEC1 signal transduction system.
\end{abstract}

\section{Introduction}

In clinic studies, Chinese Shenfoweikang herbs had been found to have effect on pre-malignant lesion, especially on gastric diseases [1,2]. The Shenfoweikang decoction might inhibit gastric carcinoma cell proliferation and cause tumor cell death. Apoptosis plays a crucial role in the proliferation and turnover of cells in various tumors. It has been clear that its extent is often enhanced in tumor by many anticancer drugs, such as cytotoxic drugs, hormone, or some Chinese herbal medicine [3-5]. Researches indicated that Chinese herbs could enhance apoptosis of human gastric cancer grafted in mice [5-7].

Differentiated embryo chondrocyte gene 1 (DEC1), a nuclear transcription factor, can translocate into nucleus, bind DNA and regulate related gene expression. Recent results showed that the expression of DEC1 was significantly higher in the gastric cancer cells than the adjacent normal tissues [8]. JAK/STATs (signal transducers and activators of transcription) signaling pathway is closely related to cell proliferation, differentiation and apoptosis, which can lead to abnormal proliferation and malignant transformation. Research confirmed that there was a high expression of STAT3 in gastric cancer, which was closely related to TNM stage, invasion depth, lymph node metastasis and tumor grade [9]. Studies showed that the overexpression of STAT3 and 
VEGF in tumor cells can increase the microvessel density and promote the progression of gastric cancer [10].

Based on the previous studies, we presumed whether the Shenfoweikang herbs could affect the apoptotic indices of gastric cancer grafted onto mice and the expression of VEGF-STAT3- signal transduction in gastric cancer, further confirming the anti-tumor mechanism of the Chinese Shenfoweikang herbs.

\section{Material and Methods}

\section{Mice and Antibody Reagents}

Fourty 6-8 weeks old BALB/C mice (weight 18-22 g) and a mice gastric carcinoma cell line MFC were obtained from the Fourth Military Medical University. The mice were subcutaneously grafted with the MFC cells. The tumor transplantation procedure was described previously [7]. Rabbit anti-mouse DEC1, STAT3 and VEGF polyclonal antibody were purchased from Santa Cruz Biotechnology, Inc (Santa Cruz, CA, USA). EnVisionTM kits were purchased from Dako Corp (Carpinteria, CA, USA).

Drugs. Shenfoweikang decoction consists of Codonopsis pilosula (Franch) Nannf., Atractylodes macrocephala koidz, Poria cocos (Schw.) Wolf, Glycyrrhiza uralensis Fisch. The concentration of the Shenfoweikang decoction was $240 \mathrm{~g} / \mathrm{L}$.

Administration. The mice were randomly divided into 4 groups, one control and the other three experimental groups, which are assigned to receive the Shenfoweikang decoction. Each animal in the three experimental groups was given $2.0 \mathrm{~mL}, 1.0 \mathrm{~mL}$ and $0.5 \mathrm{~mL}$ of the Shenfoweikang decoction through gastric perfusion every day over a 60 -day period beginning at 1 st day after grafted. The control group received normal saline according to the same schedule. All the mice were killed at 61st day after grafted.

Assessment of Tumor Growth. The effect of therapy was assessed by two ways: (1) tumor size was measured twice a week by multiplying two perpendicular diameters. (2) tumor weight was determined immediately by electron balance after the animals were killed. The tumor tissues were fixed in $10 \%$ formalin, embeded in paraffin and cut into $5 \mu \mathrm{m}$ sections coated on the slides for staining.

Detection of Apoptosis. For detection of apoptotic cells, apoptotic indices were examined by the terminal deoxynucleotidyl transferase-mediated deoxyuridine triphosphate fluorescence nick end labeling (TUNEL) method and flow cytometry analysis. (1) TUNEL: In situ cell death detection Kit POD (Roche Applied Science, Indianapolis, USA) was used to detect the apoptotic cell. The procedures were referred to the kit protocol. The positive cells were identified, counted and analyzed under the light microscope. Non-necrotic zone was selected in the tissue section and images were sent to computer by AEC camera (ZEISS, Jena, Germany). 10 image at least 1000 cells were selected on the screen, positive ratio analyzed by KS400 Video Image Digital Analysis System (ZEISS, Jena, Germany). (2) Flow cytometry analysis: Propidium iodide (PI) staining was used for flow cytometry detection of apoptosis. $10^{6}$ cells from each of the sample were treated with RNase and stained with PI. The apoptotic cells having DNA strand breaks that had been labeled were measured on a flow cytometer (FACSCalibur, Becton Dickinson, USA). The data from 106 cells/sample were collected, stored, and analyzed using CELLQUEST (Becton Dickinson USA) and ModFIT LT for mac V1.01 software (Becton Dickinson, USA).

Immunostaining Methods. All sections were deparaffinized and rehydrated with graded alcohols. Endogenous peroxidase was then blocked with $3 \mathrm{~mL} / \mathrm{L} \mathrm{H}_{2} \mathrm{O}_{2}$ diluted in methanol for $30 \mathrm{~min}$ at room temperature. Antigen retrieval was performed by 
treating the slides in citrate buffer in a microwave for $10 \mathrm{~min}$. The slides were incubated in a moist chamber with DEC1, STAT3 or VEGF rabbit polyclonal antibody $(1: 100)$ at $4^{\circ} \mathrm{C}$ overnight. After a complete wash in phosphate buffered saline (PBS), the slides were incubated with horseradish peroxidase labeled goat anti-mouse antibody (1:100) for $45 \mathrm{~min}$ at $37^{\circ} \mathrm{C}$. After a complete wash in PBS, the slides were developed in $0.5 \mathrm{~g} / \mathrm{L}$ freshly prepared 3,3'-diaminobenzedine solution (DAB, Sigma Co, St.Louis, Mo, USA) for $8 \mathrm{~min}$, and then counterstained with hematoxylin, dehydrated, air dried, and mounted. Normal mouse $\operatorname{IgG}$ was used to substitute for the primary antibody as a negative control. Only distinctive intranuclear or intra-cytoplasm immunoreactivity was considered positive. In each case, more than 1000 cells were counted and the percentage of immunoreactivity was independently determined. Image $\mathrm{J}$ analysis software was used to acquire the optical density data from the staining sections.

Statistical Analysis. All data expressed as means \pm S.D. The Student's $t$ test was performed to analyze the significance of differences in different groups of mice. $P<0.05$ was considered statistically significant.

\section{Results}

\section{Inhibition of Tumor Growth by Shenfoweikang Herbs}

Compared with the control group, tumor growth (size and weight) was significantly inhibited by treatment with the Shenfoweikang decoction $(P<0.05$, Table 1$)$. The results showed that the higher the concentration of Shenfoweikang herbs, the less the tumor weight and size. There was a significant difference between the Chinese herbs and control group.

Table 1 Chinese Shenfoweikang herbs inhibited the growth of gastric cancer $(\mathrm{x} \pm \mathrm{s})$

\begin{tabular}{|c|c|c|}
\hline Treatment & Tumor weight(g) & Tumor size $\left(\mathrm{mm}^{3}\right)$ \\
\hline High-dose Shenfoweikang Decoction & $0.48 \pm 0.32^{\mathrm{a}}$ & $257.44 \pm 36.43^{\mathrm{a}}$ \\
\hline Middle-dose Shenfoweikang Decoction & $0.64 \pm 0.25^{b}$ & $334.32 \pm 32.42^{b}$ \\
\hline Low-dose Shenfoweikang Decoction & $0.82 \pm 0.37^{\mathrm{c}}$ & $452.56 \pm 27.66^{\mathrm{c}}$ \\
\hline Saline & $2.03 \pm 0.26$ & $588.42 \pm 34.23$ \\
\hline
\end{tabular}

Induction of Tumor Cell Apoptosis by Shenfoweikang Herbs. Apoptotic index (AI) in mice loaded with gastric cancer cells was significantly elevated to $18.63 \pm 2.45 \%$ by TUNEL method and $16.86 \pm 5.63 \%$ FACScan in the Shenfoweikang decoction treatment group, compared with the controls (TUNEL: $2.88 \pm 1.62 \%$, $P<0.05$; FACScan: $5.67 \pm 2.32 \%, P<0.05$ ). But there was no significant difference between low-dose Shenfoweikang decoction treated group and control group by using either TUNEL method or flow cytometry analysis (Table 2). 
Table 2 Chinese Shenfoweikang herbs-induced apoptosis on gastric cancer cells $(\mathrm{x} \pm \mathrm{s})$

\begin{tabular}{|c|c|c|}
\hline \multirow{2}{*}{ Treatment } & \multicolumn{2}{|c|}{ Apoptotic index (AI)(\%) } \\
\cline { 2 - 3 } & TUNEL & FACScan \\
\hline High-dose Shenfoweikang Decoction & $18.63 \pm 2.45^{\mathrm{a}}$ & $16.86 \pm 5.63^{\mathrm{a}}$ \\
\hline Middle-dose Shenfoweikang Decoction & $10.76 \pm 7.45^{\mathrm{b}}$ & $9.46 \pm 4.25^{\mathrm{b}}$ \\
\hline Low-dose Shenfoweikang Decoction & $3.02 \pm 1.63^{\mathrm{c}}$ & $5.39 \pm 2.45^{\mathrm{c}}$ \\
\hline Control & $2.88 \pm 1.62$ & $5.67 \pm 2.32$ \\
\hline
\end{tabular}

${ }^{\mathrm{a}} P<0.05,{ }^{\mathrm{b}} P<0.05,{ }^{\mathrm{c}} P>0.05$ vs control group.

Expression of VEGF, STAT3 and DEC1 in Gastric Cancers. DEC1, STAT3 and VEGF immunoreactivities were detected in 40 mice gastric tumors. DEC1 proteins were mainly presenting a nuclei staining, while STAT3 and VEGF mainly stained in the cell cytoplasm. The optical density (OD) value of DEC1, STAT3 and VEGF in Shenfoweikang herbs treated groups were significantly lower than that in control $(P<$ 0.05 , Table 3).

Table 3 Expression of VEGF, STAT3 and DEC1 in gastric cancers $(\mathrm{x} \pm \mathrm{s})$

\begin{tabular}{|c|c|c|c|}
\hline \multirow{2}{*}{ Treatment } & \multicolumn{2}{|c|}{ Optical density (OD) } \\
\cline { 2 - 4 } & VEGF & STAT3 & DEC1 \\
\hline High-dose Shenfoweikang Decoction & $0.020 \pm 0.034^{\mathrm{a}}$ & $0.024 \pm 0.015^{\mathrm{a}}$ & $0.015 \pm 0.011^{\mathrm{a}}$ \\
\hline $\begin{array}{c}\text { Middle-dose Shenfoweikang } \\
\text { Decoction }\end{array}$ & $0.025 \pm 0.022^{\mathrm{b}}$ & $0.036 \pm 0.012^{\mathrm{b}}$ & $0.022 \pm 0.012^{\mathrm{b}}$ \\
\hline $\begin{array}{c}\text { Low-dose Shenfoweikang Decoction } \\
\text { Control }\end{array}$ & $0.047 \pm 0.015^{\mathrm{c}}$ & $0.055 \pm 0.023^{\mathrm{c}}$ & $0.038 \pm 0.010^{\mathrm{c}}$ \\
\hline & $0.048 \pm 0.024$ & $0.056 \pm 0.016$ & $0.039 \pm 0.015$ \\
\hline
\end{tabular}

${ }^{\mathrm{a}} P<0.05,{ }^{\mathrm{b}} P<0.05,{ }^{\mathrm{c}} P>0.05$ vs control group.

\section{Discussion}

Gastric carcinoma is one of the most common malignant gastrointestinal carcinoma in the world. At present gastric carcinoma is still detected later in most patients throughout the world, and even with curative resection, they remain at a high risk of relapse and mortality. Thus, there is a great need for effective adjuvant therapy for patients with gastric carcinoma. Our previous clinic studies suggested that Chinese herbal recipe Shenfoweikang have therapeutic effects on gastric pre-malignant lesion, with increasing the reversal of the atrophic gastritis, decreasing the recurrence and improving the life quality $[1,2]$. Because of its lower toxic side-effect compared with chemical therapy, it is worth to make a further research on its anti-cancer mechanism.

Similar to the other malignant tumors, gastric carcinoma is always accompanying with abnormal cell proliferation and differentiation. In the present study, we found that after treated with Shenfoweikang herbs, the growth of tumor were inhibited compared with the control group. We presumed that the herbs could activate the immune cells to attack the tumor cells as well as induce the apoptosis of the tumor cells directly [11-13]. The results showed that Shenfoweikang herbs could activate the immune cells to secret cytokines, perforin and granzyme B, which are able to induce the apoptosis and necrosis of tumor cells. We also found that splenocytes were activated and elicited the definite cytotoxic effects on tumor cells, which were likely to secret cytokines, such as perforin and granzyme B to exert their immune effects. It has been verified that specific immune cells could induce the apoptosis of tumor cells [14].

Apoptosis is a complex, tightly regulated, and active cellular process by which 
individual cells are triggered to undergo programmed cell death, and simultaneously will not injury neighboring cells or elicit any inflammatory reactions $[14,15]$. Various triggering factor initiate corresponding proteo-lysis cascade reaction depending on mitochondrion or APO-1/FAS/CD95 receptor mediate apoptotic pathways $[15,16]$. There are many oncogenes and tumor suppressor gene products in the regulation and execution of apoptosis. The results suggest that the mechanism of the inhibition of gastric cancer cells in vivo by Shenfoweikang herbs is related with activating immune cells and further inducing apoptosis. The VEGF-STAT3-DEC1 signal transduction pathways play an important role in the development and progression of gastrointestinal cancers. Our results suggested that Shenfoweikang herbs could down-regulate the expression of VEGF-STAT3-DEC1 signal transduction proteins in gastric cancer, thus inhibiting the growth of tumor cells.

In conclusion, Shenfoweikang decoction inhibited gastric cancer cell growth. The anti-tumor effect of Shenfoweikang herbs lies in inducing gastric cancer cells apoptosis and down-regulating the expression of VEGF-STAT3-DEC1 signal transduction protein in tumor cells. The detailed molecular mechanism of Shenfoweikang herbs inhibiting gastric cancer cells still needs further investigation.

\section{Conclusions}

Shenfoweikang herbs inhibited gastric cancer cell growth. The anti-tumor effect of Shenfoweikang herbs lies in the induction of apoptosis on gastric cancer cells and down-regulation of the expression of VEGF-STAT3-DEC1 signal transduction protein in tumor cells.

\section{Acknowledgements}

This work is supported by the Scientific Research Program of Shaanxi Provincial Education Department (No.2007JK233, 2010JK484, 14JS025), Shaanxi Administration of Traditional Chinese Medicine (No.15-SCJH001, JCPT001) and Natural Science Basic Research Plan in Shaanxi Province of China (No.2016JM8023, 2016JM8150).

\section{References}

[1] D.F. Schafer, M.F. Sorrell, Hepatocellular carcinoma, Lancet, 353 (1993) 1253-1257.

[2] Z.Y. Tang, Hepatocellular carcinoma--cause, treatment and metastasis, World J. Gastroenterol. 7 (2001) 445-454.

[3] R. Wong, C. Frenette, Updates in the management of hepatocellular carcinoma, Gastroenterol. Hepatol. (NY). 7 (2011) 16-24.

[4] P. Hanke, C. Rabe, M. Serwe, S. Böhm, C. Pagenstecher, T. Sauerbruch, W.H. Caselmann, Cirrhotic patients with or without hepatocellular carcinoma harbour AFP-specific T-lymphocytes that can be activated in vitro by human alpha-fetoprotein, Scand. J. Gastroenterol. 37 (2002) 949-955.

[5] J.A. Marrero, K.S. Henley, The role of serum biomarkers in hepatocellular carcinoma surveillance, Gastroenterol. Hepatol. (N Y). 7 (2011) 821-823.

[6] J.H. Zhong, H. Li, L.Q. Li, X.M. You, Y. Zhang, Y.N. Zhao, J.Y. Liu, B.D. Xiang, 
G.B. Wu, Adjuvant therapy options following curative treatment of hepatocellular carcinoma: a systematic review of randomized trials, Eur. J. Surg. Oncol. 38 (2012) 286-295.

[7] H.P. Lin, S.C. Li, Protective effects of Shenfoweikang on acute gastric mucous jnjury on rat, Shaanxi Journal of Traditional Chinese Medicine 28 (2007) 1422-1423. (In Chinese)

[8] Y.F. Jia, D.J. Xiao, X.L. Ma, Y.Y. Song, R. Hu, Y. Kong, Y. Zheng, S.Y. Han, R.L. Hong, Y.S. Wang, Differentiated embryonic chondrocyte-expressed gene 1 is associated with hypoxia-inducible factor $1 \alpha$ and Ki67 in human gastric cancer, Diagn. Pathol. 8 (2013) 37.

[9] P. Wu, D. Wu, L. Zhao, L. Huang, G. Shen, J. Huang, Y. Chai, Prognostic role of STAT3 in solid tumors: a systematic review and meta-analysis, Oncotarget 7 (2016) 19863-19883.

[10] J.H. Choi, M.J. Ahn, C.K. Park, H.X. Han, S.J. Kwon, Y.Y. Lee, I.S. Kim, Phospho-Stat3 expression and correlation with VEGF, p53, and Bcl-2 in gastric carcinoma using tissue microarray, APMIS. 114 (2006) 619-625.

[11] S.C. Li, F. Wang, H.P. Lin, X.Y. Zhou, Effects of Shenfoweikang on the secretion of serum gastrin and motilin in chronic atrophic gastritis, Jiangxi Journal of Traditional Chinese Medicine 38 (2007) 68-69. (In Chinese)

[12] A.G. Zhao, H.L. Zhao, X.J. Jin, J.K. Yang, L. Tang, Effects of Chinese Jianpi herbs on cell apoptosis and related gene expression in human gastric cancer grafted onto nude mice, World J. Gastroenterol. 8 (2002) 792-796.

[13] J. Li, G.Z. Sun, H.S. Lin, Y.X. Pei, X. Qi, C. An, The herb medicine formula "Yang Wei Kang Liu" improves the survival of late stage gastric cancer patients and induces the apoptosis of human gastric cancer cell line through Fas/Fas ligand and Bax/Bcl-2 pathways, Int. Immunopharmacol. 8 (2008) 1196-1206.

[14] A. Ashkenazi, V.M. Dixit, Death Receptors: Signaling and Modul ation, Science 281 (1998) 1305-1308.

[15] D.R. Green, Apoptotic pathways: the roads to ruin, Cell 94 (1998) 695-698.

[16] M. Muller, S. Wilder, D. Bannasch, D. Israeli, K. Lehlbach, L.W. Min, p53 activates the CD95 (APO-1/Fas) gene in response to DNA damage by anticancer drugs, J. Exp. Med. 188 (1998) 2033-2045. 